\title{
Cerebral blood flow autoregulation during intracranial hypertension: a simple, purely hydraulic mechanism?
}

\author{
Harold Portnoy
}

Published online: 17 January 2009

(C) Springer-Verlag 2008

Anile et al. present a study in which intracranial pressure (ICP) is elevated by progressively infusing Ringer's lactate solution until carotid artery blood flow stops. They demonstrate that the intracranial pulse pressure (pICP) first increases until blood flow begins to fall and then pICP decreases until the point where the flow stops. The reverse occurs when the infusate stops.

We have previously (author references: 13, 14, 26, 27) indicated that the mechanism is a bit different than that suggested by the authors. I agree with the authors that a critical mechanism is the Starling resistor of the bridging veins, and an autoregulatory process that compensates to maintain cerebral blood flow (CBF). We believe that the pulse wave passes from the cerebral arteries through the arterioles to the capillaries to the veins (which contain most of the cerebral blood volume) and then through the bridging veins into the dural sinuses. The venous pulse wave is transmitted though the thin flexible venous walls to the

This commentary refers to the article doi:10.1007/s00381-008-0749-7.

H. Portnoy $(\bowtie)$

Michigan Head and Spine Institute,

44200 Woodward Avenue Suite 112,

Pontiac, MI 48341, USA

e-mail: hportnoy@mhsi.us cerebrospinal fluid (CSF; the measured venous pulse being virtually identical to the CSF pulse, see above references). I believe, unlike the authors, that autoregulation is not just a compensatory increase in "upstream vascular pressure," but rather includes progressive dilation of the arterioles that results in an increase in pulse amplitude in the veins and thus the CSF pulse (pICP) until blood flow falters.

The authors have beautifully demonstrated that when CBF starts to decrease so does the pICP. Why? I would suggest that the mechanism is progressive venous compression in which the veins flatten out (because the venous lumen goes from round to flat, observing the surface of the veins may give a false impression about the lumen) [1]. As the veins progressively collapse, pulse transfer to the CSF likewise diminishes.

I am confident that the authors will continue their efforts to define the mechanism of autoregulation, and I look forward to their future researches (that may prove me wrong).

\section{Reference}

1. Wright RD (1938) Experimental observations on increased intracranial pressure. Aust N Z J Surg 7:215-235 